MEI, II, Vol. 4, nº 6, pág. 5

\title{
RePEc o cómo proveer servicios de valor añadido a una comunidad de investigadores
}

MEI

II, vol. 4

$n^{\circ} 6$

\author{
José Manuel Barrueco
}

Universitat de València

\section{Resumen}

Se describe RePEc (Research Papers in Economics), uno de los repositorios temáticos más antiguos y con más contenido de los existentes. Se presta especial atención a los servicios de valor añadido que ofrece y que convierten a RePEc en una iniciativa única, con una gran aceptación dentro de su disciplina. Finalmente se hace una breve referencia al modelo de negocios que se utiliza y que se basa en el trabajo voluntario de miles de personas de todo el mundo.

Recibido el 05-07-2013

Aceptado en 23-09-2013

\section{Palabras clave}

Repositorios temáticos, Economía, Repec, Servicios, Comunidad investigadora.

RePEc and how to provide value-added services a community of researchers

Abstract

We describe RePec (Research Papers in Economics), one of the oldest subjet repositories and with more contens as well. Special attention is paid to the addes value services offered that make RePec a unique initiative with great acceptance within their discipline. Finally there is a brief reference to the business mdel used which is based on the voluntary work of thousands of people around the world.

Keywords

Subject repository, Economics, Repec, Services, Research comunity.

\section{1.- Introducción}

Cuando se intenta sistematizar los tipos de repositorios es habitual hacer una distinción entre temáticos e institucionales. Los primeros se caracterizan por ser reducidos en número pero muy grandes en cuanto a contenidos, por haber sido los primeros en desarrollarse, por ocupar los primeros puestos en los rankings de repositorios o por ser los que gozan de mayor éxito entre la comunidad científica internacional. Mientras los segundos no han parado de crecer en los últimos años, sus contenidos son escasos, atraen poco interés por de los investigadores y están faltos de agregadores verdaderamente útiles que ofrezcan un punto de acceso único a las colecciones.

Entre los primeros se encuentra RePEc (Research Papers in Economics) $)^{1}$, la iniciativa que es objeto de este trabajo y en la que el autor del mismo colabora activamente desde su creación. En concreto, con el desarrollo de un índice de citas que se describirá en la sección tres. 
Con más de un millón de documentos almacenados, siete millones de documentos descargados el pasado año y ocupando el cuarto lugar en el mundo del Ranking Web of Repositories ${ }^{2}$, es uno de los repositorios de referencia a nivel internacional y una fuente de información básica para la comunidad investigadora en Economía.

En este artículo vamos a analizar las características que diferencian RePEc de otras iniciativas de repositorios similares intentando establecer puntos fuertes que puedan ayudar a otras instituciones a mejorar sus resultados y a ser más visibles dentro de su comunidad de investigadores.

En primer lugar, es necesario matizar una serie de puntos para entender el funcionamiento de RePEc:

- Primero, no es un repositorio en sentido estricto puesto que no almacena el texto completo de los documentos. No es un depósito de trabajos científicos sino un data set de metadatos sobre documentos que están alojados en servidores departamentales, institucionales, etc.

- Segundo, es una iniciativa descentralizada que funciona con reglas propias. La institución que desea unirse a RePEc debe utilizar su propio formato de metadatos, denominado $\mathrm{ReDIF}^{3}$, y su propio protocolo de comunicación, denominado Protocolo de Guildford 4 . Ambos preceden a otras normas más conocidas y utilizadas en la actualidad como Dublin Core u OAI-PMH respectivamente. Las normas establecidas por RePEc se han construido sobre el principio de la extrema sencillez. Cualquiera puede implementarlas sin necesidad de una inversión adicional en software o personal. La prueba de ello es que más de 1400 instituciones de todo el mundo lo han hecho.

- Tercero, todos los datos generados por RePEc (metadatos sobre documentos, citas, referencias, autores, etc.) está disponible de forma libre para ser reutilizados por cualquiera.

- Cuarto, RePEc ha tratado desde el primer momento de crear una comunidad de usuarios, por lo tanto ha sido creada por economistas para economistas, teniendo siempre presentes las características de la disciplina, las necesidades de los investigadores, la provisión de servicios útiles, el enriquecimiento de la colección mediante las contribuciones de todos los miembros de la comunidad y la reutilización de los datos, etc. En definitiva, es la comunidad quien construye RePEc. Son los cientos de voluntarios que trabajan a pequeña escala los que permiten que esta iniciativa pueda subsistir prácticamente sin financiación.

Además de estos puntos que diferencian RePEc de otras iniciativas, quizás el principal factor de éxito es la provisión de servicios de valor añadido que la convierten en una herramienta de gran utilidad para la comunidad. Esto contrasta con la escasa utilidad que aún tienen los repositorios institucionales y los agregadores de contenidos en otras disciplinas. 
Por este motivo, el objeto del presente artículo es la descripción de los servicios de valor añadido que proporciona RePEc y las posibilidades y requisitos necesarios para poder ser implementados en otras disciplinas.

\section{2.- Una breve descripción de RePEc.-}

RePEc nació en 1997 en Guildford (Reino Unido) de la mano de Thomas Krichel y a partir de una idea de Bill Goffe ${ }^{5}$, quien el 15 de Julio de 1995 escribió:

"What I would suggest is this: a distributed system with any number of sites, each mirroring each other. It would have extensive bibliographic functions (cross-referencing, etc.), and my favorite, digital timestamps for when the papers were put up. For archives outside it, papers could be listed, but no cross-referencing. But, such archives could "join" the system (say it was written in perl so could run on NT as well as Unix). Then you'd have the best of both worlds: distributed, anybody could join, extensive crossreferencing, the whole works. Such a system could easily grow with the profession's use of the net. Such a system would GREATLY benefit the profession."

La idea apuntada por Goffe de tener un sistema distribuido con muchas funcionalidades bibliográficas para beneficio de la profesión es la que ha guiado la construcción y desarrollo de RePEc en sus 16 años de vida.

En la búsqueda por reunir el mayor data set de metadatos sobre la disciplina, RePEc ha llegado a almacenar metadatos sobre 1,4 millones de documentos, de los cuales 1,2 millones tienen una versión electrónica. Los principales tipos de documentos son los artículos (850.000 ítems) y los documentos de trabajo o preprints (520.000 ítems). Ahora bien, no todos ellos son de libre acceso, mientras prácticamente la totalidad de los segundos están en abierto, los primeros proceden fundamentalmente de editoriales como Elsevier o Springer y por lo tanto son de pago. Ahora bien, para cada ítem se relacionan todas sus versiones, con lo que es muy frecuente que junto con la información del trabajo publicado aparezca una versión preprint en abierto.

El resto de tipos documentales se completan con libros, capítulos, y aplicaciones de software (65.000 ítems).

Los metadatos sobre todos estos documentos los proporcionan las instituciones que publican los mismos. En este momento hay más de 1.400 instituciones, procedentes de 76 países que contribuyen con datos a RePEc. Entre ellas están grandes editoriales como Elsevier, Wiley o Springer que publican miles de trabajos al mes, pero también pequeños departamentos universitarios que publican unos pocos preprints en su colección de documentos de trabajo. Igualmente se incluyen organizaciones internacionales como OCDE, IMF, World Bank, etc.

Todos los textos de los documentos se mantienen de forma local en la institución que los produce, lo único que se transmite a través de RePEc son los metadatos. En este sentido, RePEc funciona como un agregador de contenidos de multitud de 
repositorios institucionales, a través de un formato de metadatos y de un protocolo propios. Sobre este conjunto de datos se construyen los servicios que describimos a continuación.

\section{3.- Servicios RePEc.-}

\section{1.- Motores de búsqueda}

El primer servicio implementado fue el de motores de búsqueda. Se trata de, un servicio que se encarga de agregar los contenidos dispersos en los 1.400 proveedores de datos y organizarlos para su presentación a los investigadores. Dado que los datos de RePEc están en abierto cualquiera puede implementar un servicio de este tipo e incluso puede haber varios proporcionando funcionalidades similares y compitiendo por prestar un mejor servicio a la comunidad. De esta forma, tenemos dos motores de búsqueda: IDEAS $^{6}$ y EconPapers ${ }^{7}$. En cierta forma son la imagen de RePEc, es a través de ellos cómo los usuarios acceden a la práctica totalidad de las características que analizaremos más adelante y que han sido integradas en los mismos.

Ambos proporcionan funcionalidades similares: acceso a todos los metadatos de RePEc, organización mediante motor de búsquedas (simple y avanzada) complementado con una navegación por tipos de documentos o bien por códigos de la clasificación JEL (Journal of Economics Literature Classification), posibilidad de descargar, compartir y exportar datos bibliográficos, etc. Se diferencian básicamente en la apariencia en la presentación de los datos, lo que convierte en una cuestión de preferencia estética para el usuario utilizar uno u otro.

Según los datos de utilización, IDEAS es el que mayor cuota de mercado tiene con un $75 \%$ del total de descargas frente al $18 \%$ de EconPapers.

Analizando la serie histórica de descargas $^{8}$ vemos que estos servicios han experimentado una caída considerable en los últimos tres años. Si bien la tendencia es a estabilizarse, el número de descargas en 2012, lejos de incrementarse como sería de esperar al aumentar el número de documentos disponibles, se sitúa al nivel de 2007. Esto se debe a que Google Scholar no ofrece como resultados de las búsquedas los documentos procedentes de RePEc, puesto que no tienen detrás una versión PDF. Es decir que Google prioriza a los repositorios institucionales o las páginas de los productores, cuando existen, frente a los resultados procedentes de agregadores como IDEAS o EconPapers. Esto pone de manifiesto la importancia de Google como fuente de información científica y también el problema de caída en la visibilidad que está experimentando RePEc.

\section{2.- Control de autoridades e identificación de autores}

El asunto de la identificación de autores está de plena actualidad en el mundo de los repositorios y de la gestión de la investigación en general como lo demuestra la abundante literatura existente al respecto o el lanzamiento de iniciativas como $\mathrm{ORCID}^{9} \mathrm{O} \mathrm{ISNI}^{10}$. 
Básicamente, el problema es cómo asociar una persona física a un código o cadena de caracteres bajo el cual agrupar toda su producción científica así como datos adicionales como contacto, lugar de trabajo, etc. Es lo que en bibliotecas llamaríamos un control de autoridades, pero aumentado con datos complementarios.

Este problema ya está solucionado en el ámbito de RePEc desde hace más de diez años a través del RePEc Author Service (RAS) ${ }^{11}$. Es un servicio de registro de autores en el que a cada individuo que se registra se le asigna un código, se le pide que identifique todas las variantes de su nombre que ha utilizado (el sistema le presenta una lista de posibilidades para facilitarle la tarea), que identifique toda su producción disponible en RePEc y aporte datos de contacto sobre las instituciones en las que trabaja.

A cambio de este registro el autor recibe toda una serie de servicios adicionales, que serán tanto más completos cuanta mejor sea la información que ha proporcionado sobre sí mismo. De ahí que haya un incentivo claro para el autor en detallar todos los datos que se solicitan.

Entre los servicios que se ofrecen tenemos: página personalizada en los buscadores de RePEc con su producción en un único lugar; inclusión en los rankings que veremos más adelante, datos actualizados mensualmente sobre el uso (visualizaciones y descargas) y las citas recibidas por sus documentos, etc.

Existen más de 36.000 investigadores registrados en RAS.

\section{3.- Archivo huérfano}

La contribución de metadatos sobre publicaciones en RePEc nunca se pensó como algo que se pudiera hacer de forma particular sino canalizada a través de las instituciones productoras. Esto genera el problema de cómo dar cabida a publicaciones procedentes de investigadores cuyas instituciones no contribuyen a RePEc, bien por falta de interés, porque tienen una temática que es marginal a la Economía o simplemente porque no tienen ninguna colección de publicaciones.

Para solucionarlo se creó el Munich Personal RePEc Archive (MPRA) ${ }^{12}$ gestionado por la biblioteca de la universidad de Múnich. Se trata de un repositorio temático, gestionado con el software e-prints, donde puede remitir documentos que recojan resultados de investigación en Economía, cualquier persona. Los documentos son revisados por un grupo de editores voluntarios, cada uno de ellos encargado de una lengua diferente. La política de remisión de trabajos es bastante sencilla y se resume en cinco puntos, se aceptan trabajos:

- de naturaleza académica,

- relacionados con cualquier aspecto de la Economía,

- que sean originales,

- que aporten contenidos completos, no se aceptan resúmenes, 
- cuyos derechos de propiedad intelectual estén en manos del autor.

El éxito de este archivo ha sido considerable, alcanzando los casi 25.000 documentos cargados por los autores y convirtiéndose así en un repositorio temático más.

\section{4.-Análisis de uso}

Todos los datos de utilización de los servicios RePEc son centralizados en un servidor encargado de proporcionar estadísticas sobre visualizaciones y descargas de documentos. Este servidor se denomina $\log \mathrm{Ec}^{13}$.

Mensualmente los servicios remiten a LogEc los logs generados por sus servidores web. En LogEc se les aplica un procedimiento de limpieza ${ }^{14}$ destinado a tratar de separar las consultas y descargas realizadas por robots y otras aplicaciones de las realizadas por personas físicas que son las realmente interesantes. Igualmente, se eliminan los denominados doble clics y se aplican procesos para detectar descargas automáticas y diferentes abusos.

Tras el proceso de limpieza más del 75\% de las conexiones se han eliminado. Sobre el $25 \%$ restante es sobre el que se trabaja para la provisión de datos estadísticos a dos niveles: autores y documentos. Cada autor registrado en RAS tiene una página en LogEc con su producción y datos históricos de visualizaciones y descargas desde 1998. De cada documento se ofrece lo mismo, una serie histórica con visualizaciones y descargas desde que ese documento entró a formar parte de RePEc.

Además de los datos en bruto, el verdadero potencial de las estadísticas se puede obtener cuando se comparan los datos o se hace una explotación de los mismos. Las herramientas de explotación que proporciona LogEc son los rankings. Se pueden ver por defecto, rankings de los documentos más descargados por su tipología, los autores y las revistas. Además, se proporciona una herramienta para configurar los propios rankings en función de criterios geográficos, por ejemplo, podemos obtener los $\mathrm{X}$ investigadores más descargados en España.

En general, los rankings son una herramienta que estimula la competencia y la participación. Este es un servicio muy bien acogido por los investigadores quienes suelen estar impacientes por conocer los últimos datos, lo que se plasma en multitud de mensajes cuando la edición de los datos se retrasa algún día.

Demostrar a los investigadores que sus documentos se usan debería ser una prioridad dentro del ecosistema de los repositorios. Si bien a título individual todos los repositorios institucionales están proporcionando estos datos, lo que verdaderamente tiene sentido es que dichos datos se agreguen para permitir comparaciones. RECOLECTA ${ }^{15}$ en España debería ser quien se encargara de proporcionar estos datos en nuestro país. Unos datos que potencialmente y según establece la Ley de la Ciencia, podrían ser utilizados con fines de evaluación. 


\section{5.- Alertas sobre novedades}

Un servicio tradicional en nuestras bibliotecas siempre ha sido el de proporcionar alertas sobre novedades. Eso es precisamente lo que hace NEP (New Economic Papers $)^{16}$.

Se trata de un servicio de alertas que utiliza principalmente el correo electrónico y de forma secundaria los RSS. Funciona a través de más de 90 listas de distribución, cada una de ellas especializada en un área temática (contabilidad, bancos centrales, desarrollo, econometría, etc.) Cada lista tiene un editor que colabora de forma voluntaria que se encarga de seleccionar semanalmente las novedades en su área y remitirlas en un mensaje a los subscriptores de su lista. Es así una alerta que proporciona el valor añadido de un control de calidad, puesto que no se distribuye todo, sino solamente lo que el editor considera que es interesante para sus lectores. Los mensajes se guardan además en la página web y se distribuyen como RSS.

En total, casi 33.000 personas, es decir, direcciones diferentes de correo, están registrados en NEP.

Proporcionar un servicio de alertas agregado entre todos los repositorios institucionales es otro servicio que debería ser candidato a ser prestado por RECOLECTA. No es interesante para un investigador saber lo nuevo que se publica en su institución sino lo que se publica en su disciplina.

\section{6.- Índice de citas}

RePEc proporciona un índice de citas elaborado de forma automática desde 2001. Se denomina CitEc (Citations in Economics) ${ }^{17}$. Es un sistema similar al que utiliza Google Citations pero que lo ha precedido en casi diez años. El análisis de citas es el complemento necesario para evaluar los resultados de investigación. Con los datos de uso sabemos cuántos investigadores se han interesado por un documento, pero las citas son el indicador de cuántos de ellos además lo han leído, les ha parecido interesante y han optado por mencionarlo en su trabajo.

Básicamente, se trata de un sistema informático que es capaz de descargar los documentos que se encuentran en libre acceso en internet, analizarlos para identificar la lista de referencias o documentos citados, extraer cada referencia, dividirla entre los elementos que la constituyen y, a partir de ellos, determinar si el documento citado está disponible en RePEc. Si lo está se considera que es una cita y se crea el enlace correspondiente.

En estos momentos, se han procesado para extraer las referencias más de 500.000 documentos, es decir algo más del 33\% de los disponibles en RePEc. De ellos se han extraído 12,5 millones de referencias y casi 5 millones de citas.

Como se puede comprobar por el porcentaje de documentos procesados queda mucho por hacer. Entre los problemas para incrementar este número, el más 
importante es la falta de acceso. Si el texto no está en libre acceso y no existe acuerdo de colaboración con el proveedor, el sistema no puede procesarlo. En otras ocasiones el problema viene porque el proveedor no ha dado un enlace al texto sino a una página intermedia desde la que el usuario lo descarga. Esto funciona con humanos pero es más complejo de automatizar.

Por estas razones desde hace dos años, además de continuar mejorando el sistema informático, se ha pasado a buscar la colaboración de los investigadores y editores:

Tenemos acuerdos con editores como Elsevier para que nos proporcionen en formato XML la información detallada de las referencias de cada artículo que publican. En otros casos permiten la descarga controlada de PDFs desde el número IP de la máquina que hace CitEc.

Determinar cuándo una referencia se refiere a un documento en RePEc es una cuestión de comparación, de ver cómo de parecida es la referencia a los metadatos que nos constan en RePEc. Por este motivo el detectar o no una cita depende de donde se ponga el límite en la comparación. El límite para el sistema está colocado bastante alto, lo cual significa que hay pocos falsos positivos, es decir, citas que realmente no lo son. Pero para aquellas referencias que no se está tan seguro, se utiliza RAS y la colaboración de los autores para identificarlas. Así el autor a través de RAS puede ver una lista de sugerencias de citas y aceptar o denegar las que considere. Estos datos son luego integrados con CitEc.

Por otro lado, cualquiera puede completar las citas de un documento. Ante una lista de referencias, aquellos elementos que el sistema no ha encontrado se les coloca un enlace "Add citation now". Esto significa: nosotros pensamos que el documento que representa esta referencia no está en RePEc, pero si estamos equivocados, por favor, indícanos el identificador del documento citado. Los datos remitidos por esta vía son revisados por el gestor de CitEc para comprobar que son auténticos.

También, cualquier autor registrado en RAS puede aportar la lista de referencias de un documento que no ha podido ser procesado automáticamente. Para ello, se le proporciona una ventana donde puede copiar y pegar la lista de referencias para que sea procesada.

A partir de los datos recopilados se ofrecen perfiles de citas para cada documento, serie o autor registrado en RAS. Son unos datos que están disponibles para cualquiera que desee hacer una implementación de los mismos. Por ejemplo, los motores de búsqueda de los que hablamos antes añaden a la descripción de los documentos dos elementos extraídos de CitEc: citado por y cita a.

Es de particular interés el perfil del autor puesto que en una página se ofrece toda su producción con las citas de cada obra así como una serie de indicadores como su índice $\mathrm{H}$, autores con los que colabora, etc. 


\section{7.- Rankings de economistas ${ }^{18}$}

Ya hemos mencionado lo importante que son los rankings para estimular la colaboración dentro de una comunidad. Conscientes de ellos, cada autor registrado en RAS puede entrar a participar en los rankings de economistas.

Tomando como base los datos de producción científica, citas, visualizaciones y descargas se combinan para crear una serie de indicadores que después se organizan en forma de ranking. Entre ellos, factor de impacto simple y recursivo, índice H, etc.

Junto con los rankings habituales de autores, instituciones y documentos, se ofrecen otros más curiosos como jóvenes economistas, economistas fallecidos, mujeres economistas, etc.

Los rankings son a menudo utilizados para fines de evaluación por parte de departamentos e instituciones económicas.

\section{8.- Otros servicios menos académicos}

Además de los descritos anteriormente existen otros servicios de menor contenido académico, así por ejemplo:

- RePEc Genealogy ${ }^{19}$, intenta determinar dentro del campo de la Economía y de los autores registrados en RAS, quién fue alumno de quién, cuándo y dónde. Es decir que se trata de establecer relaciones del tipo: autor A fue alumno de autor B, se graduó en institución I en el año Y. Hasta el momento el 18\% de los autores registrados han contribuido su genealogía desde Septiembre de 2012.

- RePEc Biblio ${ }^{20}$ o bibliografía básica. Partiendo de una estructura jerárquica de temas o materias, cada autor puede añadir las lecturas que considera imprescindibles para su área de interés. Un editor, voluntario, se encarga de revisar, en la medida de lo posible, los contenidos que se envían.

- RePEc reading lists o bibliografía recomendada dentro de alguna asignatura de los estudios de Economía.

\section{9.- Otros sitios que utilizan los datos RePEc pero no forman parte de la iniciativa}

Dado que todos los datos están en abierto y pueden reutilizarse por cualquiera, es frecuente que agregadores de contenidos los utilicen para crear sus servicios. Si nos atenemos a los principios de RePEc cada institución que quisiera hacer uso de los materiales debería:

- No cobrar por los datos ni incluirlos en productos que no sean de libre acceso.

- En la visualización de los documentos debe especificarse, al menos: Título, Autor y restricciones en el acceso si las tuviera. 
- Contribuir a enriquecer los datos de RePEc, por ejemplo, compartiendo los logs de accesos y descargas.

- Respetar los acuerdos de copyright que pudieran existir en otros proveedores de datos participantes en RePEc.

- Indicar la procedencia de los datos incluyendo el logo de RePEc.

No obstante, en la práctica no siempre se cumplen estos puntos. Entre los sitios que utilizan datos de RePEc pero no cumplen alguno de los principios enumerados tenemos: EconLit, WorldCat, Scirus, Sciverse, etc... Es difícil saber cuántos existen puesto que no tienen relación directa con RePEc.

\section{4.- Modelo de negocios}

Una última característica que diferencia RePEc de otras iniciativas es su modelo de negocios. Mientras en otros casos es necesaria una financiación para que una o varias instituciones lleven a cabo el servicio, por ejemplo, la Universidad de Cornell y arXiv, en RePEc se confía en la comunidad lo que permite funcionar con un presupuesto de cero euros.

Ahora bien, que no haya presupuesto no quiere decir que no haya gastos. El único gasto necesario en RePEc es el mantenimiento de los servidores, puesto que el resto de trabajo se aporta de forma voluntaria por los colaboradores, como se ha descrito en el punto anterior. Para obtener financiación para sus servidores se adopta un modelo de esponsorización. Cada servicio RePEc con sus correspondientes máquinas están patrocinados por una institución determinada. Así NEP está patrocinado por Victoria University of Wellington, CitEc por INOMICS, IDEAS por el Federal Reserve Bank of Saint Louis, etc.

Históricamente, la institución se hacía cargo del hosting de una máquina física y su conexión a internet, pero actualmente se ha optado por un almacenamiento en la nube por ser más barato y efectivo que el local. El alcance de este patrocinio varía pero en general no supera los 1.000 euros anuales.

\section{5.- Conclusiones}

En este trabajo se ha descrito RePEc desde el punto de vista de los servicios de valor añadido que presta a la comunidad. Creemos que uno de los factores de éxito de esta iniciativa son precisamente estos valores que la convierten en una herramienta de gran utilidad para los investigadores.

Es de esperar que veamos aparecer servicios como los descritos aquí en los agregadores de contenidos de los repositorios institucionales como RECOLECTA. Si queremos que los repositorios se conviertan en una pieza más del sistema de comunicación científica es necesario que sean percibidos como algo útil y valioso por parte de los investigadores. Los bibliotecarios ya estamos convencidos de ello, ahora falta convencer a los usuarios. 
MEI, II, Vol. 4, nº 6, pág. 15

\section{6.- Notas}

1. http://repec.org

2. http://repositories.webometrics.info/en/world

3. http://ideas.repec.org/p/rpc/rdfdoc/redif.html

4. http://ideas.repec.org/p/rpc/rdfdoc/guildp.html

5. http://ideas.repec.org/e/pgo 13.html

6. http://ideas.repec.org

7. http://econpapers.repec.org

8. http://logec.repec.org

9. http://orcid.org

10. http://isni.org

11. http://authors.repec.org

12. http://mpra.repec.org

13. http://logec.repec.org

14. http://logec.repec.org/about.htm\#stats

15. http://recolecta.net

16. http://nep.repec.org

17. http://citec.repec.org

18. http://ideas.repec.org/top

19. http://genealogy.repec.org

20. http://biblio.repec.org 